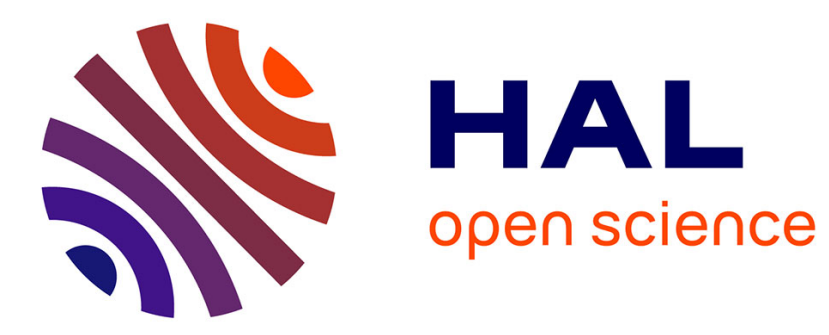

\title{
Statistical mechanics of a colloidal suspension in contact with a fluctuating membrane
}

\author{
Thomas Bickel, Mabrouk Benhamou, Hamid Kaïdi
}

\section{To cite this version:}

Thomas Bickel, Mabrouk Benhamou, Hamid Kaïdi. Statistical mechanics of a colloidal suspension in contact with a fluctuating membrane. Physical Review E: Statistical, Nonlinear, and Soft Matter Physics, 2004, 70, pp.051404. hal-00002731

\section{HAL Id: hal-00002731 \\ https://hal.science/hal-00002731}

Submitted on 31 Aug 2004

HAL is a multi-disciplinary open access archive for the deposit and dissemination of scientific research documents, whether they are published or not. The documents may come from teaching and research institutions in France or abroad, or from public or private research centers.
L'archive ouverte pluridisciplinaire HAL, est destinée au dépôt et à la diffusion de documents scientifiques de niveau recherche, publiés ou non, émanant des établissements d'enseignement et de recherche français ou étrangers, des laboratoires publics ou privés. 


\title{
Statistical mechanics of a colloidal suspension in contact with a fluctuating membrane
}

\author{
T. Bickel用 \\ Centre de Physique Moléculaire Optique et Hertzienne, \\ Université Bordeaux 1, UMR 5798, \\ 351 cours de la Libération, 33405 Talence, France \\ M. Benhamou and H. Kaïdi \\ Laboratoire de Physique des Polymères et Phénomènes Critiques, \\ Faculté des Sciences Ben M'sik, B.P. 7955, Casablanca, Morocco
}

(Dated: August 31, 2004)

\begin{abstract}
Surface effects are generally prevailing in confined colloidal systems. Here we report on dispersed nanoparticles close to a fluid membrane. Exact results regarding the static organization are derived for a dilute solution of non-adhesive colloids. It is shown that thermal fluctuations of the membrane broaden the density profile, but on average colloids are neither accumulated nor depleted near the surface. The radial correlation function is also evaluated, from which we obtain the effective pairpotential between colloids. This entropically-driven interaction shares many similarities with the familiar depletion interaction. It is shown to be always attractive with range controlled by the membrane correlation length. The depth of the potential well is comparable to the thermal energy, but depends only indirectly upon membrane rigidity. Consequenses for stability of the suspension are also discussed.

PACS numbers: 05.20.-y, 82.70.Dd, 87.16.Dg
\end{abstract}

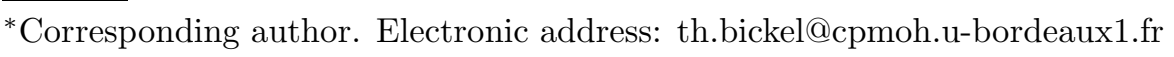




\section{INTRODUCTION}

Fluid membranes are soft surfaces, self-assembled from surfactant solutions [1]. They assume a large variety of shapes and topologies, which have been accurately explained in terms of bending energy [2, 3]. In most practical realizations, however, membrane suspensions are not pure but incorporate colloidal entities as well. In living systems for instance, lipid bilayers organize the cell into compartments that keep apart different chemical environments 6]. Biological membranes are therefore in contact with various kinds of proteins, macro-ions or more complex structures [由]. Membrane phases used in detergency or cosmetics also include numerous additives, like macromolecules or colloids, in order to improve efficiency and to control viscoelastic properties [5]. One is then naturally inclined to investigate transformations that may occur upon addition of colloids, and several studies have recently been devoted to these complex systems. Essentially, these have focused on the softening of membrane resulting from the depletion of spherical and rod-like colloids [7], or the depletion [8, 9] and adsorption [10, 11, 12] of flexible polymers, but the predicted effects are usually small compared to the bare rigidity of the bilayer. Bending of a membrane upon colloid adsorption has also drawn growing interest because of potential applications for drug encapsulation and gene delivery [13, 14, 15, 16, 17]. Nevertheless, a common feature shared by most theoretical studies is that membrane fluctuations are systematically disregarded. This point clearly illustrates the technical difficulty to couple bulk and surface degrees of freedom.

Generally, the mutual influence of bulk and surface properties on each other is a challenging problem [18, 19]. The situation has now been clarified for bidispersed hard-sphere suspensions [20, 21, 22]: when in contact with a flat substrate, excluded-volume effects are known to push the larger beads toward the wall of the sample [23]. Recent experiments done with curved or corrugated surfaces have shown that geometric features of the surface can also create and modulate entropic force fields [24, 25]. These depletion forces can be used to grow oriented colloidal crystal, with numerous potential applications such as the fabrication of photonic bandgap crystals [26]. The theoretical description of those systems usually requires advanced density functional techniques [21], that have been adapted to study depletion potentials close to arbitrarily shaped substrates [27]. In this work, we follow a different line and present some new findings regarding the static organization of nanopar- 
ticles near a fluctuating surface. Given the increasing complexity of the problem, we focus on the simplest system consisting of a monodispersed, dilute solution of non-adhesive colloids. This allows us to derive exact results and to highlight non-trivial phenomena, such as membrane-induced interaction between the colloids.

This paper is organized as follows. In Sec. II), we present an exact computation of the partition function of the global system. The determination of the variation of the particle density upon distance is the aim of Sec. III. The computations of the radial distribution function and the resulting pair-potential and related discussion are presented in Sec. IV. We draw some concluding remarks in the last section. Finally, some technical details are relegated to the appendices.

\section{THE PARTITION FUNCTION}

Consider a fluctuating membrane in contact with a colloidal suspension, consisting of nanoparticles immersed in some solvent. We assume that the colloids cannot permeate through the bilayer on experimental time-scales, in such way that the membrane act as a flexible but impenetrable wall for the particles. In this paper, we shall use the notation $\mathbf{r}=(\boldsymbol{\rho}, z)$, where $\boldsymbol{\rho}=(x, y)$ is the transverse vector and $z$ the perpendicular component. The position of the (almost flat) membrane is specified through the displacement field $h(x, y)$. The surface fluctuates around the horizontal plane $z=0$, so that the height $h(x, y)$ may take either positive or negative values. The equilibrium statistical mechanics of membranes is based on the Helfrich Hamiltonian [2]

$$
\mathcal{H}_{0}[h]=\frac{1}{2} \int d^{2} \boldsymbol{\rho}\left[\kappa(\Delta h)^{2}+\sigma(\nabla h)^{2}+\mu h^{2}\right],
$$

where $(\kappa, \sigma, \mu)$ are the elastic constants of the membrane. In what follows, we shall rather use the rescaling parameters: $\widehat{\kappa}=\beta \kappa, \widehat{\sigma}=\beta \sigma$, and $\widehat{\mu}=\beta \mu$, where we define as usually $\beta=1 / k_{B} T$, with $T$ the absolute temperature and $k_{B}$ the Boltzmann constant. Let us also introduce the height-height correlation function

$$
G\left(\boldsymbol{\rho}-\boldsymbol{\rho}^{\prime}\right)=\left\langle h(\boldsymbol{\rho}) h\left(\boldsymbol{\rho}^{\prime}\right)\right\rangle_{0}-\langle h(\boldsymbol{\rho})\rangle_{0}\left\langle h\left(\boldsymbol{\rho}^{\prime}\right)\right\rangle_{0},
$$

from which one obtains the mean-squared fluctuations $\xi_{\perp}^{2}=G(0)$. Some properties of $G$ are recalled in Appendix A. 
We intend to compute some statistical properties of the system under investigation, namely the particle density profile and radial distribution function [28]. For a dilute solution, one may ignore the interactions between particles and treat them as an ideal gas in confined geometry. Since we are interested in those systems containing a considerable number of particles (thermodynamic limit), the physical quantities are independent of the particular choice of statistical ensemble. For convenience, we shall consider both the canonical and the grand canonical ensembles. The grand canonical partition function, denoted $\mathcal{Z}_{G}$, is the Laplace transform of the canonical partition function, that is

$$
\mathcal{Z}_{G}=\sum_{N=0}^{+\infty} f^{N} \mathcal{Z}_{c}(N)
$$

with $f$ the fugacity. In the above equality, the canonical partition function $\mathcal{Z}_{c}(N)$ is

$$
\mathcal{Z}_{c}(N)=\frac{1}{\lambda^{3 N} N !} \int d^{3} \mathbf{r}_{1} \ldots d^{3} \mathbf{r}_{N} \int \mathcal{D} h e^{-\beta \mathcal{H}[h]} e^{-\beta \sum_{i=1}^{N} V\left(\mathbf{r}_{i}\right)} .
$$

The functional integral extends over all configurations of the field $h$, weighted with the Helfrich energy (1). The positions $\mathbf{R}_{N}=\left(\mathbf{r}_{1}, \ldots, \mathbf{r}_{N}\right)$ of the $N$ particles are restricted to the upper side of the three-dimensional space limited by the membrane. In the above equation, the thermal wavelength $\lambda$ results from integration over the particles momenta, and colloidmembrane interactions are accounted for through the (contact) hard core potential $V(\mathbf{r})$

$$
V\left(\mathbf{r}_{i}\right)= \begin{cases}0, & \text { if } z_{i}>h\left(x_{i}, y_{i}\right) \\ +\infty, & \text { otherwise }\end{cases}
$$

With these considerations, we first examine the canonical partition function. It can be written

$$
\mathcal{Z}_{c}=\frac{\mathcal{Z}_{0}}{\lambda^{3 N} N !} \int \prod_{i=1}^{N} d^{3} \mathbf{r}_{i} \varphi_{N}\left(\mathbf{R}_{N}\right)
$$

where it is convenient to define the function $\varphi_{N}$

$$
\varphi_{N}\left(\mathbf{R}_{N}\right)=\mathcal{Z}_{0}^{-1} \times \int \mathcal{D} h e^{-\beta \mathcal{H}[h]} \prod_{i=1}^{N} \theta\left[z_{i}-h\left(x_{i}, y_{i}\right)\right] .
$$

Here, $\mathcal{Z}_{0}=\int \mathcal{D} h \exp \{-\beta \mathcal{H}[h]\}$ is the partition function of a membrane in the absence of particles $(N=0)$, and $\theta(x)$ is the step function. It is easy to see, from its definition (7), that $\varphi_{N}$ satisfies the following boundary conditions at infinity

$$
\begin{aligned}
& \left.\varphi_{N}\left(\boldsymbol{\rho}_{1}, \ldots, \boldsymbol{\rho}_{N} ; z_{1}, \ldots, z_{N}\right)\right|_{z_{1}=\ldots=z_{N}=-\infty}=0, \\
& \left.\varphi_{N}\left(\boldsymbol{\rho}_{1}, \ldots, \boldsymbol{\rho}_{N} ; z_{1}, \ldots, z_{N}\right)\right|_{z_{1}=\ldots=z_{N}=+\infty}=1
\end{aligned}
$$


for fixed values of the transverse vectors $\left(\boldsymbol{\rho}_{1}, \ldots, \boldsymbol{\rho}_{N}\right)$. The function $\varphi_{N}\left(\mathbf{R}_{N}\right)$ has to be understood as the partition function of a membrane whose configurations are subjected to $N$ restrictions $h\left(x_{i}, y_{i}\right) \leq z_{i}$, with $1 \leq i \leq N$. It is generally a complicated function of the relative transverse distances $\left|\boldsymbol{\rho}_{i}-\boldsymbol{\rho}_{j}\right|$ and perpendicular components $z_{i}$. Indeed, the translation symmetry is preserved in the parallel directions, but due to the presence of the membrane, this symmetry is broken in the perpendicular one. After some algebra detailed in Appendix B, we find that $\varphi_{N}$ is given by

$$
\varphi_{N}\left(\mathbf{R}_{N}\right)=(2 \pi)^{-N / 2}\left[\operatorname{det} \mathcal{G}_{N}\right]^{-1 / 2} \int_{-\infty}^{z_{1}} d z_{1}^{\prime} \ldots \int_{-\infty}^{z_{N}} d z_{N}^{\prime} \exp \left\{-\frac{1}{2} \sum_{i, j=1}^{N} z_{i}^{\prime} \cdot\left[\mathcal{G}_{N}^{-1}\right]_{i j} . z_{j}^{\prime}\right\},
$$

where $\mathcal{G}_{N}$ is the squared matrix of order $N$ whose elements are the propagators $\left[\mathcal{G}_{N}\right]_{i j}=$ $G\left(\boldsymbol{\rho}_{i}-\boldsymbol{\rho}_{j}\right)$ defined in Eq. (2). This expression is compatible with the boundary conditions (8) and (9), and more generally we can deduce from Eq. (10) the fundamental property according to which

$$
0 \leq \varphi_{N}\left(\boldsymbol{\rho}_{1}, \ldots, \boldsymbol{\rho}_{N} ; z_{1}, \ldots, z_{N}\right) \leq 1
$$

for all values of the position vectors $\left(\mathbf{r}_{1}, \ldots, \mathbf{r}_{N}\right)$. The right-hand side inequality relies on the fact that $\varphi_{N}$ is an incomplete Gaussian multiple-integral.

Now, we direct our attention to the grand canonical function. Formally, it writes as

$$
\begin{aligned}
\mathcal{Z}_{G} & =\sum_{N=0}^{+\infty} f^{N} \frac{1}{\lambda^{3 N} N !} \int \mathcal{D} h e^{-\beta \mathcal{H}[h]} \int \prod_{i=1}^{N} d^{3} \mathbf{r}_{i} \theta\left[z_{i}-h\left(\boldsymbol{\rho}_{i}\right)\right] \\
& =\int \mathcal{D} h e^{-\beta \mathcal{H}[h]} \sum_{N=0}^{+\infty} f^{N} \frac{1}{\lambda^{3 N} N !}\left\{\int d^{3} \mathbf{r} \theta[z-h(\boldsymbol{\rho})]\right\}^{N} \\
& =\int \mathcal{D} h e^{-\beta \mathcal{H}[h]} \exp \left\{\frac{f}{\lambda^{3}} \int d^{3} \mathbf{r} \theta[z-h(\boldsymbol{\rho})]\right\}
\end{aligned}
$$

Integrating over the $z$-variable then yields

$$
\mathcal{Z}_{G}=\mathcal{Z}_{0} e^{f \Omega / 2 \lambda^{3}} \times \frac{\int \mathcal{D} h e^{-\beta \mathcal{H}[h]-f \lambda^{-3} \int d^{2} \boldsymbol{\rho} h(\boldsymbol{\rho})}}{Z_{0}} .
$$

Here, $\Omega=S \times L$ is the total volume occupied by the system under investigation, $S$ is the area of the horizontal plane and $L / 2$ represents the upper bound of the perpendicular coordinate $z$. The above functional integral can be easily calculated, and we find

$$
\frac{\int \mathcal{D} h e^{-\beta \mathcal{H}[h]-f \lambda^{-3} \int d^{2} \boldsymbol{\rho} h(\boldsymbol{\rho})}}{\mathcal{Z}_{0}}=\exp \left\{f^{2} S \widetilde{G}(0) / 2 \lambda^{6}\right\}
$$


with $\widetilde{G}(0)=1 / \widehat{\mu}$ the Fourier transform of the propagator $G$ at $q=0$. To obtain this result, we have applied the general formula (B5) of Appendix $B$ to the particular source $J(\boldsymbol{\rho})=-f \lambda^{-3}$. Finally, we have the simple, exact expression for the partition function $\mathcal{Z}_{G}$

$$
\mathcal{Z}_{G}=\mathcal{Z}_{0} \times \exp \left\{\frac{f \Omega}{2 \lambda^{3}}\right\} \times \exp \left\{\frac{f^{2} S}{2 \widehat{\mu} \lambda^{6}}\right\} .
$$

Noticeably, $\mathcal{Z}_{G}$ splits into three parts. The first two factors are the standard contributions: $\mathcal{Z}_{0}$ is the partition function of the membrane in a particle-free solvent, and $\exp \left\{f \Omega / 2 \lambda^{3}\right\}$ is the partition function of an ideal gas of colloids (remember that the particles are restricted to the upper half of the space). All the information concerning the interplay between bulk and surface contributions finally factorizes in the last term of Eq. (15).

With this expression of $\mathcal{Z}_{G}$, we are now able to evaluate the average number of colloids

$$
\langle N\rangle=f \frac{\partial \ln \mathcal{Z}_{G}}{\partial f}=\frac{f \Omega}{2 \lambda^{3}} \times\left(1+\frac{2 f}{\widehat{\mu} L \lambda^{3}}\right)
$$

In addition to the usual term $f \Omega / 2 \lambda^{3}$, we find a second contribution that happens to be negligible at low concentration $(f<<1)$ or for very large perpendicular extension $\left(L>>\widehat{\mu}^{-1} \lambda^{-3}\right)$. For the sake of simplicity, we will assume thereafter that these requirements are fulfilled so that the bulk concentration is

$$
\rho_{\infty}=\frac{\langle N\rangle}{\Omega / 2} \simeq f \lambda^{-3}
$$

Note, however, that the second contribution in Eq. (16) could not be disregarded in a strongly confining system. This point might be relevant for experimental realizations involving colloids in a lamellar phase [29, 30] or in a sponge phase [31] of membranes.

\section{PARTICLE DENSITY PROFILE}

The concentration profile of an ideal gas of colloids in contact with a rigid wall located at $z=0$ is simply $\rho_{H W}=\rho_{\infty} \times \theta(z)$. For a flexible interface, the situation is quite different as thermal undulations are expected to broaden the distribution. In this section, we examine the mean-value of the particle density at point $\mathbf{r}$ defined by

$$
\rho(\mathbf{r})=\left\langle\sum_{i=1}^{N} \delta_{3}\left(\mathbf{r}-\mathbf{r}_{i}\right)\right\rangle=N\left\langle\delta_{3}\left(\mathbf{r}-\mathbf{r}_{1}\right)\right\rangle,
$$


with $\delta_{3}$ the three-dimensional Dirac distribution. The averaging procedure implies integration over the colloid configurations as well as internal degrees of freedom of the membrane. Explicitly, we have

$$
\begin{aligned}
\rho(\mathbf{r}) & =\frac{1}{\mathcal{Z}_{G}} \sum_{N=0}^{+\infty} \frac{f^{N}}{\lambda^{3 N} N !} N \int \mathcal{D} h e^{-\beta \mathcal{H}[h]} \int \prod_{i=1}^{N-1} d^{3} \mathbf{r}_{i} \theta\left[z_{i}-h\left(x_{i}, y_{i}\right)\right] \theta[z-h(\boldsymbol{\rho})] \\
& =\frac{f \lambda^{-3}}{\mathcal{Z}_{G}} \int \mathcal{D} h e^{-\beta \mathcal{H}[h]} \exp \left\{\frac{f}{\lambda^{3}} \int d^{3} \mathbf{r}^{\prime} \theta\left[z^{\prime}-h\left(\boldsymbol{\rho}^{\prime}\right)\right]\right\} \theta[z-h(\boldsymbol{\rho})] \\
& =\mathcal{Z}_{0} \frac{f \lambda^{-3}}{\mathcal{Z}_{G}} e^{f \Omega / 2 \lambda^{3}} \times \frac{\int \mathcal{D} h e^{-\beta \mathcal{H}[h]-f \lambda^{-3} \int d^{2} \boldsymbol{\rho}^{\prime} h\left(\boldsymbol{\rho}^{\prime}\right)} \theta[z-h(\boldsymbol{\rho})]}{\mathcal{Z}_{0}} .
\end{aligned}
$$

The last functional integral can be computed making a simple translation of the field $h \rightarrow$ $h+f / \lambda^{3} \widehat{\mu}$. We finally get

$$
\rho(\mathbf{r})=f \lambda^{-3} \times \varphi_{1}\left(\boldsymbol{\rho} ; z+f / \lambda^{3} \widehat{\mu}\right)
$$

where $\varphi_{1}$ is a particular function of type (10), that is

$$
\varphi_{1}\left(\boldsymbol{\rho} ; z+f / \lambda^{3} \widehat{\mu}\right)=[2 \pi G(0)]^{-1 / 2} \int_{-\infty}^{z+f / \lambda^{3} \widehat{\mu}} d z^{\prime} \exp \left\{-\frac{1}{2} G^{-1}(0) z^{2}\right\} .
$$

For $N=1$, the squared matrix $\mathcal{G}_{1}$ in relation (10) reduces to $G(0)=\xi_{\perp}^{2}$. Recalling that $f \lambda^{-3}$ equals the bulk density $\rho_{\infty}$, we find the final expression for the density profile

$$
\rho(z)=\rho_{\infty} \times \frac{1}{2}\left[1+\operatorname{erf}\left(\frac{z+z_{0}}{\sqrt{2} \xi_{\perp}}\right)\right]
$$

with the characteristic length

$$
z_{0}=\rho_{\infty} \widehat{\mu}^{-1}
$$

The concentration profile is shown in fig. (四). As expected, it only depends on the perpendicular distance $z$ (homogeneity property in the parallel directions). For fixed parameter $\widehat{\mu}$, the scale $z_{0}$ becomes smaller as the particle density is decreased. The physical meaning of this length can be understood as follows. When in contact with the colloidal solution, the membrane experiences the osmotic pressure of the particles. At low concentration, this pressure is proportional to the concentration of particles in contact with a flat surface $p_{\text {osm }}=k_{B} T \rho_{\infty}$. Indeed, we see from Eq. (13) that integration over the colloid positions leads to an effective Hamiltonian for the membrane $\mathcal{H}_{\text {eff }}=\mathcal{H}_{0}+p_{\text {osm }} \int d^{2} \rho h(\rho)$. The average position of the membrane is then shifted to its new value $\langle h\rangle=-z_{0}$, so that the concentration profile (or the dividing surface) is translated from the same distance. For a symmetric system (particles 
on both sides with the same chemical potential), this length would just vanish. Note that when $z=-z_{0}$, the local particle density is reduced to half of the bulk value, that is $\rho_{\infty} / 2$.

Now, let us compute the excess particle density, defined as the first moment of the density profile 32

$$
\Gamma=\int_{-\infty}^{+\infty} d z\left[\rho(z)-\rho_{\infty} \times \theta\left(z+z_{0}\right)\right]
$$

$\Gamma$ is called the adsorption of the species, since a large positive value of $\Gamma$ is an evidence for particle accumulation at the surface. Conversely, a negative value of $\Gamma$ indicates that concentration in the surface vicinity is lower than concentration in the bulk phase. Using relation (22), we find after a simple integration

$$
\Gamma=0
$$

meaning that there is, on average, neither accumulation nor depletion of particles near the membrane. Actually, this result comes from the cancellation of two effects: insertion of particles into the holes and valleys of the rough surface exactly compensates for the depletion from the convex regions, as can be seen in fig. (1). Eq. (25) also implies that there is no additional contribution to the interfacial tension, and consequently no spontaneous curvature of the membrane induced by the colloids. However, this result is only valid for vanishing particle radius $a$. Although finite size effects are not easily included in the theory, one does not expect these results to hold for $a \sim \xi_{\|}$any longer 16, 33.

\section{RADIAL DISTRIBUTION FUNCTION AND EFFECTIVE POTENTIAL}

To better characterize the statistical properties of the system, we now focus on the pair distribution function defined as

$$
g\left(\mathbf{r}, \mathbf{r}^{\prime}\right)=\frac{\sum_{i \neq j}\left\langle\delta_{3}\left(\mathbf{r}-\mathbf{r}_{i}\right) \delta_{3}\left(\mathbf{r}^{\prime}-\mathbf{r}_{j}\right)\right\rangle}{\rho(\mathbf{r}) \rho\left(\mathbf{r}^{\prime}\right)} .
$$

Using the same techniques as before, we find that $g\left(\mathbf{r}, \mathbf{r}^{\prime}\right)$ can be expressed in terms of the $\varphi_{N}$ functions defined in (10). Without further detail, we obtain the formal expression

$$
g\left(\mathbf{r}, \mathbf{r}^{\prime}\right)=\frac{\varphi_{2}\left(\boldsymbol{\rho}, \boldsymbol{\rho}^{\prime} ; z+z_{0}, z^{\prime}+z_{0}\right)}{\varphi_{1}\left(\boldsymbol{\rho} ; z+z_{0}\right) \varphi_{1}\left(\boldsymbol{\rho}^{\prime} ; z^{\prime}+z_{0}\right)} .
$$


As one could expect, only $\varphi_{i}$ 's with $N=1$ and 2 come out of the calculations. For the sake of completeness, we give explicitly the radial distribution function

$$
g\left(\mathbf{r}, \mathbf{r}^{\prime}\right)=\frac{(2 \pi)^{-1}\left[\operatorname{det} \mathcal{G}_{2}\right]^{-1 / 2} \int_{-\infty}^{z+z_{0}} d z_{1} \int_{-\infty}^{z^{\prime}+z_{0}} d z_{2} \exp \left\{-\frac{1}{2}\left(z_{1}, z_{2}\right) \mathcal{G}_{2}^{-1}\left(\begin{array}{l}
z_{1} \\
z_{2}
\end{array}\right)\right\}}{\frac{1}{4}\left[1+\operatorname{erf}\left(\frac{z+z_{0}}{\sqrt{2} \xi_{\perp}}\right)\right]\left[1+\operatorname{erf}\left(\frac{z^{\prime}+z_{0}}{\sqrt{2} \xi_{\perp}}\right)\right]}
$$

where $\mathcal{G}_{2}$ is the $2 \times 2$ correlation matrix

$$
\mathcal{G}_{2}=\left[\begin{array}{ll}
G(0) & G\left(\left|\boldsymbol{\rho}-\boldsymbol{\rho}^{\prime}\right|\right) \\
G\left(\left|\boldsymbol{\rho}-\boldsymbol{\rho}^{\prime}\right|\right) & G(0)
\end{array}\right]
$$

and $\operatorname{det} \mathcal{G}_{2}=G(0)^{2}-G\left(\left|\boldsymbol{\rho}-\boldsymbol{\rho}^{\prime}\right|\right)^{2}$. Once again, the result depends on the relative transverse distance $\left|\boldsymbol{\rho}-\boldsymbol{\rho}^{\prime}\right|$, whereas it varies with $z$ and $z^{\prime}$ separately. We emphasize that this pair correlation function would be identically unity for an ideal gas confined by a rigid wall. Here, surface fluctuations give rise to bulk correlations between colloids over distances that depends both on their separation and on their upright distance from the membrane. For fixed $z$ and $z^{\prime}, g\left(\mathbf{r}, \mathbf{r}^{\prime}\right)$ is maximum when $\boldsymbol{\rho}=\boldsymbol{\rho}^{\prime}$ and decreases to 1 as the transverse separation increases. The equality $g\left(\mathbf{r}, \mathbf{r}^{\prime}\right)=1$ (no correlations) is only achieved either when $z$ or $z^{\prime}$ goes to $+\infty$ at fixed parallel distance $\left|\boldsymbol{\rho}-\boldsymbol{\rho}^{\prime}\right|$, or when $\left|\boldsymbol{\rho}-\boldsymbol{\rho}^{\prime}\right| \rightarrow+\infty$ at fixed $z$ and $z^{\prime}$. Indeed, the former requirement expresses that the colloids do not feel the surface anymore at elevations higher than $\xi_{\perp}$, whereas the latter asserts that correlations vanish at parallel separation much larger than the membrane correlation length $\xi_{\|}$(see Appendix $A$ ).

With the help of the computed one- and two-points distribution functions, we can extract the membrane-induced interactions between particles. According to Eq. (6), an effective $N-$ body potential $\mathcal{U}\left(\mathbf{r}_{1}, . ., \mathbf{r}_{N}\right)$ may be defined through

$$
\varphi_{N}\left(\boldsymbol{\rho}_{1}, . ., \boldsymbol{\rho}_{N} ; z_{1}, . ., z_{N}\right)=e^{-\beta \mathcal{U}\left(\mathbf{r}_{1}, . ., \mathbf{r}_{N}\right)}
$$

The analyze developped in this report indicates that the many-body interaction decomposes as

$$
\mathcal{U}\left(\mathbf{r}_{1}, \ldots, \mathbf{r}_{N}\right)=\sum_{i} \mathcal{U}_{1}\left(\mathbf{r}_{i}\right)+\sum_{\{i, j\}} \mathcal{U}_{2}\left(\mathbf{r}_{i}, \mathbf{r}_{j}\right)+\ldots+\mathcal{U}_{N}\left(\mathbf{r}_{1}, . ., \mathbf{r}_{N}\right)
$$

Here, $\mathcal{U}_{1}$ is the effective external potential resulting from thermal undulations of the membrane. We easily find

$$
\mathcal{U}_{1}(\mathbf{r})=-k_{B} T \ln \frac{\rho(z)}{\rho_{\infty}}
$$


As the reduced density $\rho(z) / \rho_{\infty}$ ranges from 0 to 1 , this potential is always repulsive and tends to move the particles away from the surface. Note that the corresponding force $F=-d \mathcal{U}_{1} / d z$ exerted on a particle by surface undulations remains finite at "contact": $F\left(-z_{0}\right)=k_{B} T / \xi_{\perp}$ (recall that $-z_{0}$ is the average position of the membrane under the osmotic pressure of the colloids). Surprisingly, this force increases as the roughness of the surface $\xi_{\perp}$ decreases, but it has to be this way as $F$ eventually diverges in the hard wall limit.

Regarding the potential of mean force $\mathcal{U}_{2}\left(\mathbf{r}, \mathbf{r}^{\prime}\right)$, we find

$$
\mathcal{U}_{2}\left(\mathbf{r}, \mathbf{r}^{\prime}\right)=-k_{B} T \ln g\left(\left|\boldsymbol{\rho}-\boldsymbol{\rho}^{\prime}\right|, z, z^{\prime}\right)
$$

The normalization of $g\left(\mathbf{r}, \mathbf{r}^{\prime}\right)$, Eq. (27), ensures that only two-body terms are accounted for. The pair potential $\mathcal{U}_{2}$ is shown in fig. (2) for a membrane with no surface tension $(\sigma=0)$ and for fixed $z=z^{\prime}$ : it is negative at short parallel distances and vanishes at large separations. Accordingly, colloids that are close to the membrane tend to aggregate even if there are only hard-core repulsions in our description. We can evaluate the depth of this potential: diagonalizing the quadratic form in (28) leads to

$$
\mathcal{U}_{2}\left(\mathbf{r}=\mathbf{r}^{\prime}\right)=-k_{B} T \ln 2+k_{B} T \ln \left[1+\operatorname{erf}\left(\frac{z+z_{0}}{\sqrt{2} \xi_{\perp}}\right)\right]
$$

Of course, the interaction still depends on the $z$-position of the pair of colloids. The depth of the potential increases with decreasing altitude, and is of order $k_{B} T$ for $z=-z_{0}$. At larger separation, $\mathcal{U}_{2}$ displays a tiny repulsive barrier $\left(0.01 k_{B} T\right.$ at most). In the limit $d=\left|\boldsymbol{\rho}-\boldsymbol{\rho}^{\prime}\right| \gg \xi_{\|}$, we show in Appendix $C$ that the potential of mean force vanishes exponentially

$$
\mathcal{U}_{2}(d) \sim k_{B} T \sqrt{\frac{\xi_{\|}}{d}} e^{-d / \xi_{\|}}
$$

for $z=z^{\prime}=z_{0}$.

\section{DISCUSSION}

In this paper, we have adapted the usual many-body statistical mechanics in order to include an additional degree of freedom, namely the thermal fluctuations of the membrane. It has been shown that surface undulations broaden the density profile and generates correlations among an essentially ideal gas system. As can be seen in Eq. (13), partial integration 
over the positions induces a linear coupling with the membrane height in the case of pointlike particles. For finite-size objects, one would certainly expect further couplings with membrane curvature as well as with higher-order terms, but this point is far beyond the scope of this paper.

As a consequence of surface fluctuations, the colloids attract each other through the potential of mean force $\mathcal{U}_{2}$. Interstingly, the only remaining signature of the elastic parameters of the membrane are the length scales $\xi_{\|}, \xi_{\perp}$ and $z_{0}$. For two particles at a given position, the configurational entropy of the membrane increases as the colloids come closer. This effective pair interaction is of order of the thermal energy for particles near the average position of the membrane, and is in many respects similar to the familiar depletion interaction. In particular, it would simply sum up with a direct colloid-colloid potential in a more realistic system. Note that our approach is to some extent peculiar, in the sense that we trace out the degrees of freedom of the "slow" variable, ending up with an effective Hamiltonian for the small particles. This procedure usually leads to a very poor description of the system, because one has generally to resort to uncontrolled approximations. Here, the situation is more refined as we managed to perform exact calculations. The potential of mean force (33) is therefore expected to be very accurate for colloids much smaller than the correlation length $\xi_{\|}$.

Finally, we would like to emphasize the fact that $\mathcal{U}_{1}$ and $\mathcal{U}_{2}$ are the dominant interactions at low concentrations. The framework developed in this paper would in principle allow us to evaluate the relative weight of many-body contributions, but these would only be relevant at the onset of an hypothetical aggregation. Here however, the drift force from the membrane always move the particle away from the surface so that two-body attraction is not prevailing. One could still imagine to enforce particle accumulation near the membrane through a small attractive colloid-membrane interaction. Whether membrane fluctuations could then induce surface cristallization is an interesting point, work on this question is currently under progress.

\section{Acknowledgments}

The authors are much indebted to Professors C. Misbah and G. Porte for illuminating discussions during the Spring School on Soft-Matter Physics and its Applications, 12-16 
April 2004, Marrakech, Morocco.

\section{APPENDIX A}

In this appendix, we recall a couple of results on fluid membranes. The height-height correlation function is defined as

$$
\begin{aligned}
G(\boldsymbol{\rho}) & =\langle h(\boldsymbol{\rho}) h(0)\rangle_{0}-\langle h(\boldsymbol{\rho})\rangle_{0}\langle h(0)\rangle_{0} \\
& =\int \frac{d^{2} \mathbf{q}}{(2 \pi)^{2}} \frac{e^{i \mathbf{q} \cdot \boldsymbol{\rho}}}{\widehat{\kappa} q^{4}+\widehat{\sigma} q^{2}+\widehat{\mu}},
\end{aligned}
$$

with the notation $q=|\mathbf{q}|$. Here, the thermal averages $\langle\ldots\rangle_{0}$ are performed with the Helfrich Hamiltonian (1) in the absence of particle. For a bilayer without surface tension $(\sigma=0)$, the integral over the Fourier modes leads to

$$
G(\rho)=-\frac{4}{\pi} \xi_{\perp}^{2} \operatorname{kei}\left(\sqrt{2} \frac{\rho}{\xi_{\|}}\right),
$$

with $\operatorname{kei}(x)=\operatorname{Im}\left[K_{0}\left(x e^{i \pi / 4}\right)\right]$ is a Kelvin function [34]. We then define the mean roughness of the membrane $\xi_{\perp}=G(0)^{1 / 2}=2^{-3 / 2}(\widehat{\kappa} \cdot \widehat{\mu})^{-1 / 4}$, and the in-plane correlation length $\xi_{\|}=$ $2^{1 / 2}(\widehat{\kappa} / \widehat{\mu})^{1 / 4}$ characterizing the exponential decay of $G(\rho)$ at large distances

$$
G(\rho) \sim e^{-\rho / \xi_{\|}} \quad, \quad \rho>>\xi_{\|}
$$

\section{APPENDIX B}

The aim of this appendix is the proof of formula (10) that defines the function $\varphi_{N}$. To this end, we first compute its multiple derivative

$$
\frac{\partial^{N} \varphi_{N}}{\partial z_{1} \ldots \partial z_{N}}=\mathcal{Z}_{0}^{-1} \times \int \mathcal{D} h e^{-\beta \mathcal{H}[h]} \prod_{j=1}^{N} \delta\left[z_{j}-h\left(x_{j}, y_{j}\right)\right]
$$

Write the integral form of the Dirac distribution

$$
\delta\left[z_{j}-h\left(x_{j}, y_{j}\right)\right]=\int_{-\infty}^{+\infty} \frac{d k_{j}}{2 \pi} e^{i k_{j} \cdot\left[z_{j}-h\left(x_{j}, y_{j}\right)\right]},
$$

allows us to rewrite Eq. (B1) as

$$
\frac{\partial^{N} \varphi_{N}}{\partial z_{1} \ldots \partial z_{N}}=\int_{-\infty}^{+\infty} \prod_{j=1}^{N}\left[\frac{d k_{j}}{2 \pi} e^{i k_{j} . z_{j}}\right] \times \frac{\int \mathcal{D} h e^{-\beta \mathcal{H}[h]+\int d^{2} \boldsymbol{\rho} J(\boldsymbol{\rho}) h(\boldsymbol{\rho})}}{\mathcal{Z}_{0}}
$$


Here, we have introduced the source

$$
J(\boldsymbol{\rho})=-i \sum_{j=1}^{N} k_{j} \delta_{2}\left(\boldsymbol{\rho}-\boldsymbol{\rho}_{j}\right)
$$

with the two-dimensional vector $\boldsymbol{\rho}_{j}=\left(x_{j}, y_{j}\right)$. The functional integration in relation (B3) is trivial, since the Hamiltonian $\mathcal{H}[h]$ is quadratic in the field $h$. We simply give the result

$$
\frac{\int \mathcal{D} h e^{-\beta \mathcal{H}[h]+\int d^{2} \boldsymbol{\rho} J(\boldsymbol{\rho}) h(\boldsymbol{\rho})}}{Z_{0}}=\exp \left\{\frac{1}{2} \int d^{2} \boldsymbol{\rho} \int d^{2} \boldsymbol{\rho}^{\prime} J(\boldsymbol{\rho}) G\left(\boldsymbol{\rho}-\boldsymbol{\rho}^{\prime}\right) J\left(\boldsymbol{\rho}^{\prime}\right)\right\},
$$

where $G\left(\boldsymbol{\rho}-\boldsymbol{\rho}^{\prime}\right)$ is the membrane propagator, relation (2). Now, replace the source $J(\boldsymbol{\rho})$ by its definition (B4) to find

$$
\frac{1}{2} \int d^{2} \boldsymbol{\rho} \int d^{2} \boldsymbol{\rho}^{\prime} J(\boldsymbol{\rho}) G\left(\boldsymbol{\rho}-\boldsymbol{\rho}^{\prime}\right) J\left(\boldsymbol{\rho}^{\prime}\right)=-\frac{1}{2} \sum_{j=1}^{N} k_{i} G\left(\boldsymbol{\rho}_{i}-\boldsymbol{\rho}_{j}\right) k_{j} .
$$

We therefore obtain

$$
\begin{aligned}
\frac{\partial^{N} \varphi_{N}}{\partial z_{1} \ldots \partial z_{N}} & =\int_{-\infty}^{+\infty} \prod_{j=1}^{N} \frac{d k_{j}}{2 \pi} e^{i k_{j} \cdot z_{j}} \exp \left\{-\frac{1}{2} \sum_{i, j=1}^{N} k_{i} \cdot\left[\mathcal{G}_{N}\right]_{i j} \cdot k_{j}\right\} \\
& =(2 \pi)^{-N / 2}\left[\operatorname{det} \mathcal{G}_{N}\right]^{-1 / 2} \times \exp \left\{-\frac{1}{2} \sum_{i, j=1}^{N} z_{i}^{\prime} \cdot\left[\mathcal{G}_{N}^{-1}\right]_{i j} \cdot z_{j}^{\prime}\right\} .
\end{aligned}
$$

The $N^{2}$ coefficients $\left[\mathcal{G}_{N}\right]_{i j}=G\left(\boldsymbol{\rho}_{i}-\boldsymbol{\rho}_{j}\right), 1 \leq i, j \leq N$, define a squared matrix $\mathcal{G}_{N}$. Remark that the above multiple integral is Gaussian. A straightforward integration yields the explicit expression of the function $\varphi_{N}$, formula (10).

\section{APPENDIX C}

At large transverse separation $d=\left|\boldsymbol{\rho}-\boldsymbol{\rho}^{\prime}\right| \gg \xi_{\|}$, it is possible to evaluate completely the two-points function $\varphi_{2}$. Indeed, the propagator satisfies in this limit $G(d) \ll G(0)=\xi_{\perp}^{2}$, so that we are naturally lead to define the small parameter $\alpha=G(d) / G(0)$. At first order, one has $\operatorname{det} \mathcal{G}=\xi_{\perp}^{2}\left(1+\mathcal{O}\left(\alpha^{2}\right)\right)$, and

$$
\left(z_{1}, z_{2}\right) \mathcal{G}^{-1}\left(\begin{array}{c}
z_{1} \\
z_{2}
\end{array}\right)=\frac{z_{1}^{2}}{\xi_{\perp}^{2}}+\frac{z_{2}^{2}}{\xi_{\perp}^{2}}-2 \alpha \frac{z_{1} z_{2}}{\xi_{\perp}^{2}}+\mathcal{O}\left(\alpha^{2}\right)
$$

Expanding the exponential in Eq. (10) up to first order, we find

$$
\begin{aligned}
\varphi_{2}\left(d, z, z^{\prime}\right) & =(2 \pi)^{-1} \xi_{\perp}^{-2} \int_{-\infty}^{z+z_{0}} d z_{1} \int_{-\infty}^{z^{\prime}+z_{0}} d z_{2} \exp \left\{-\frac{z_{1}^{2}+z_{2}^{2}}{2 \xi_{\perp}^{2}}\right\}\left(1+\alpha \frac{z_{1} z_{2}}{\xi_{\perp}^{2}}+\mathcal{O}\left(\alpha^{2}\right)\right) \\
& =\varphi_{1}(z) \varphi_{1}\left(z^{\prime}\right)+\frac{G(d)}{2 \pi \xi_{\perp}^{2}} \exp \left\{-\frac{\left(z+z_{0}\right)^{2}}{2 \xi_{\perp}^{2}}\right\} \exp \left\{-\frac{\left(z^{\prime}+z_{0}\right)^{2}}{2 \xi_{\perp}^{2}}\right\}+\mathcal{O}\left(\alpha^{2}\right) .
\end{aligned}
$$


Taking the logarithm of this expression and using the definition (A2) for $G(d)$ finally leads to Eq. (35).

[1] S. Safran, Statistical Thermodynamics of Surfaces, Interfaces and Membranes (AddisonWesley, Reading, MA, 1994).

[2] W. Helfrich, Z. Naturforsch. 28c (1973) 693.

[3] For a recent review, see U. Seifert, Advances in Physics 46 (1997) 13.

[4] C. Fradin, A. Abu-Arish, R. Granek, and M. Elbaum, Biophys. J. 84 (2003) 2005.

[5] D.F. Evans and H. Wennerström, The Colloidal Domain (Wiley, New-York, 1999).

[6] H. Lodish, A. Berk, S.L. Zipursky, P. Matsudaira, D. Baltimore, and J. Darnell, Molecular Cell Biology (Freeman \& Company, New-York, 2002).

[7] K. Yaman, P. Pincus, and C.M. Marques, Phys. Rev. Lett. 78 (1997) 4514.

[8] R. Podgornik, Europhys. Lett. 21 (1993) 245.

[9] A. Hanke, E. Eisenriegler, and S. Dietrich, Phys. Rev. E 59 (1999) 6853.

[10] J.T. Brooks, C.M. Marques, and M.E. Cates, J. Phys. II (France) 1 (1991) 673.

[11] F. Clément and J.-F. Joanny, J. Phys. II (France) 7 (1997) 973.

[12] K.I. Skau and E.M. Blokhuis, Eur. Phys. J. E 7 (2002) 13.

[13] J.O. Rädler, I. Koltover, T. Salditt, and C.R. Safinya, Science 275 (1997) 810.

[14] C. Dietrich, M. Angelova, and B. Pouligny, J. Phys. II (France) 7 (1997) 1651.

[15] I. Koltover, J.O. Rädler, and C.R. Safinya, Phys. Rev. Lett. 82 (1999) 1991.

[16] R. Lipowsky and H.-G. Döbereiner, Europhys. Lett. 43 (1998) 219.

[17] M. Deserno and T. Bickel, Europhys. Lett. 62 (2003) 767.

[18] A.A. Louis, P.G. Bolhuis, E.J. Meijer, and J.-P. Hansen, J. Chem. Phys. 116 (2002) 10547.

[19] R.R. Netz and D. Andelman, Phys. Rep. 380 (2003) 1.

[20] T. Biben, P. Bladon, and D. Frenkel, J. Phys. Condens. Matter 8 (1996) 10799.

[21] R. Roth, R. Evans, and S. Dietrich, Phys. Rev. E 62 (2000) 5360.

[22] C. N. Likos, Phys. Rep. 348 (2001) 267.

[23] P.D. Kaplan, J.L. Rouke, A.G. Yodh, and D.J. Pine, Phys. Rev. Lett. 72 (1994) 582.

[24] A.D. Dinsmore, A.G. Yodh, and D.J. Pine, Nature (London) 383 (1996) 239.

[25] A.D. Dinsmore, D.T. Wong, P. Nelson, and A.G. Yodh, Phys. Rev. Lett. 80 (1998) 409. 
[26] K.-H. Lin, J.C. Crocker, V. Prasad, A. Schofield, D.A. Weitz, T.C. Lubensky, and A.G. Yodh, Phys. Rev. Lett. 85 (2000) 1770.

[27] P. Bryk, R. Roth, M. Schoen, and S. Dietrich, Europhys. Lett. 63 (2003) 233.

[28] J.-P. Hansen and I.R. McDonald, Theory of Simple Liquids (Academic Press, London, 1976).

[29] G. Bouglet and C. Ligoure, Eur. Phys. J. B 9 (1999) 137.

[30] M. Imai, R. Mawatari, K. Nakaya, and S. Komura, Eur. Phys. J. E 13 (2004) 391.

[31] H. Tanaka, M. Isobe, and J. Yamamoto, Phys. Rev. Lett. 89 (2002) 168303.

[32] J.S. Rowlinson and B. Widom, Molecular Theory of Capillarity (Clarendon Press, Oxford, 1982).

[33] T. Bickel, J. Chem. Phys. 118 (2003) 8960.

[34] M. Abramowitz and I.A. Stegun, Handbook of Mathematical Functions (Dover, New-York, 1972). 


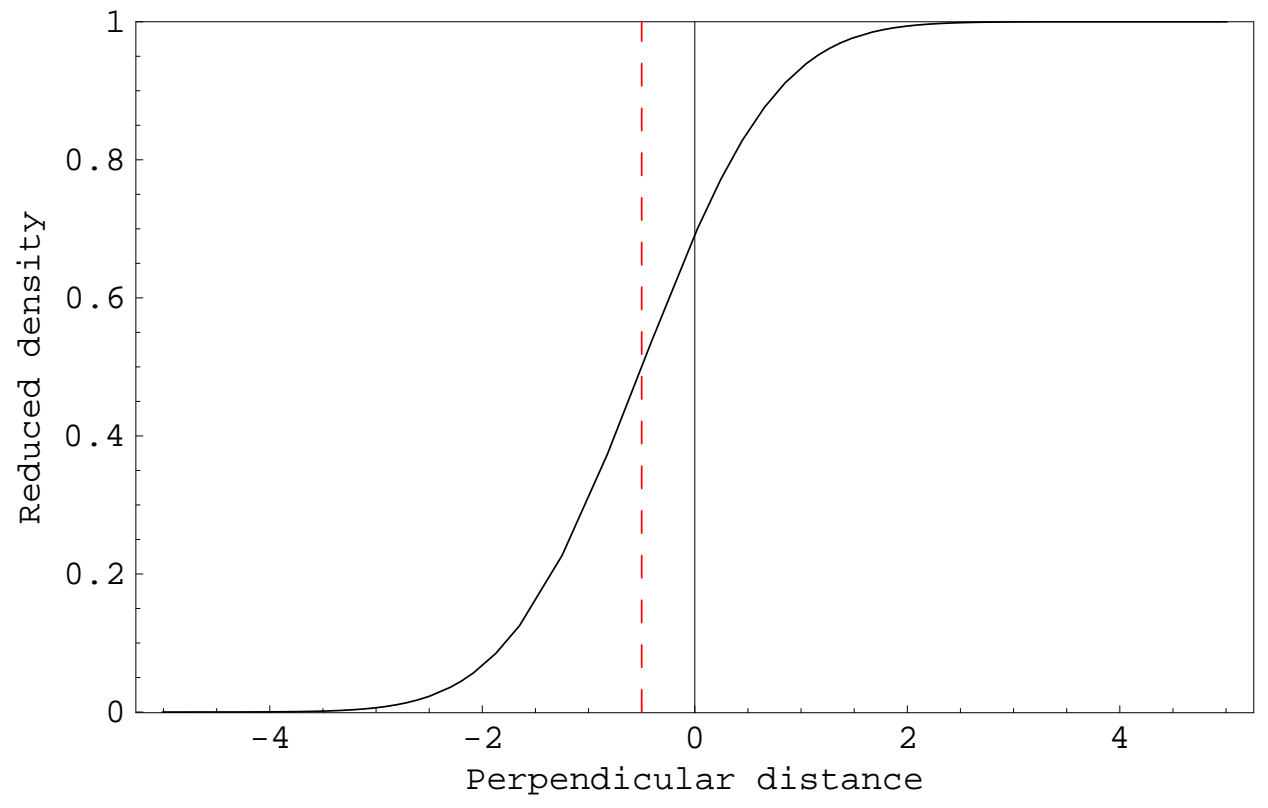

FIG. 1: Reduced density $\rho(z) / \rho_{\infty}$ as a function of the reduced perpendicular distance $z / \xi_{\perp}$. The density profile is symetric with respect of the dotted line $z=-z_{0}$, so that the number of particles that enter the depressions of the undulating surface exactly compensate for the depleted ones. 


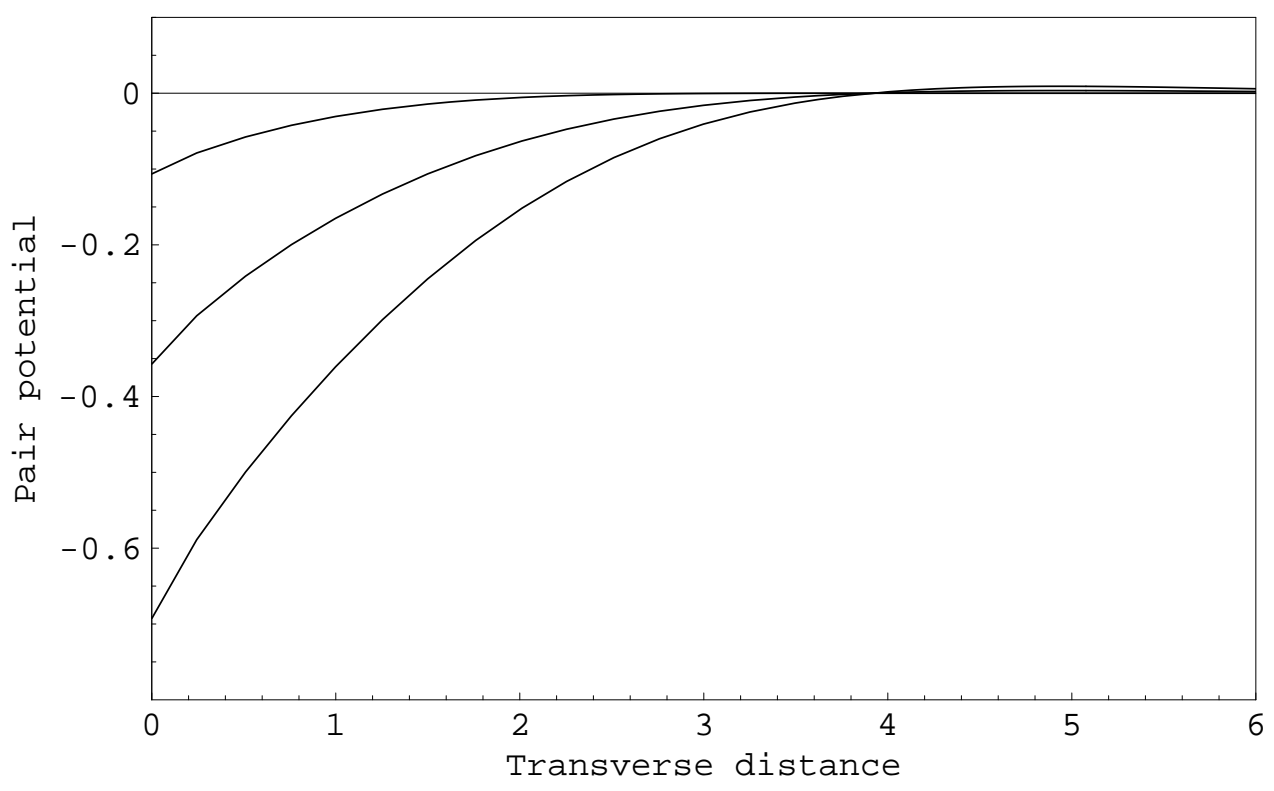

FIG. 2: Equal-height, effective pair-potential $\beta \mathcal{U}_{2}\left(\boldsymbol{\rho}, \boldsymbol{\rho}^{\prime}, z=z^{\prime}\right)$ as a function of the reduced transverse distance $\left|\boldsymbol{\rho}-\boldsymbol{\rho}^{\prime}\right| / \xi_{\|}$. The curves corresponds to different values of the perpendicular distance. From bottom to top: $\left(z+z_{0}\right) / \xi_{\perp}=0,0.5$, and 1 . The depth of the potential well is of order $k_{B} T$ for $z+z_{0}=0$, and vanishes exponentially at large separations. 